\title{
Eye wash water flow direction study: an evaluation of the effectiveness of eye wash devices with opposite directional water flow
}

This article was published in the following Dove Press journal:

Clinical Ophthalmology

\author{
Jennifer S Fogt \\ Lisa A Jones-Jordan \\ Joseph T Barr
}

The Ohio State University College of Optometry, Columbus, OH, USA
Correspondence: Jennifer S Fogt The Ohio State University College of Optometry, $338 \mathrm{~W}$ I0th Avenue, Columbus OH 43210, USA

Tel + I 6I42920882

Email fogt.78@osu.edu
Introduction: New designs of eye wash stations have been developed in which the direction of water flow from the fountain has been reversed, with two water streams originating nasally in both eyes and flowing toward the temporal side of each eye. No study has been done to determine the ideal direction of water flow coming from the eye wash in relation to the eye.

Materials and methods: Ophthalmic eye examinations were conducted before and after the use of two eye wash stations with opposite water flow directionality. Fluorescein was instilled in both eyes before using an eye wash to measure the effectiveness of the water flow. Subjects were surveyed upon their experiences using the eye washes.

Conclusion: Ophthalmic examination found no significant difference in the efficacy of the eye washes with nasal-to-temporal water flow when compared to temporal-to-nasal water flow direction.

Keywords: eye wash, ocular burn, cornea, water flow

\section{Introduction}

Chemical contact with the eye can cause severe ocular damage leading to blindness. ${ }^{1}$ Treatment of an ocular burn can be severe and difficult to manage. ${ }^{2}$ Chemical burns to the eye can occur in any setting, with young children often having accidents in the home, ${ }^{3}$ and adult males experiencing the majority of ocular chemical burn accidents in a work or industrial setting. ${ }^{2}$ An ocular burn can vary in severity depending on the amount of exposure and the type of chemical. Both acidic and alkali chemicals alter the $\mathrm{pH}$ of the eye and cause damage to the ocular surface. ${ }^{1}$ A weak acid does not penetrate the cornea well, while hydrofluoric acid can quickly penetrate the eye and cause severe injury. ${ }^{4}$ Alkali burns penetrate the cornea quickly, causing more severe damage than most acid burns. ${ }^{5}$ Chemical burns are classified into different grades of injury depending on the severity of ocular damage, with very poor visual prognosis for those with deep tissue damage. ${ }^{1}$

While it is widely accepted that eye protection, such as safety goggles would be an effective primary source of prevention, ${ }^{6}$ those who have neglected to wear protection or who have had a severe accident in which eye protection was not effective, still need to minimize the effects of chemicals if they have splashed into the eyes. Once a patient with a chemical injury presents to an emergency room, chemical neutralization is preferred to manage the toxic reactions in the ocular tissue. ${ }^{7}$ Solutions that can buffer an acid or base are preferable to simple dilution, and there has been a move for emergency personnel to utilize amphoteric and hypertonic chelating solutions to neutralize ocular and cutaneous chemical accidents, ${ }^{8}$ although these have not yet been 
widely adopted in the workplace or emergency facilities. While surgical and medical treatments exist for injured eyes after a burn, those eyes that receive immediate irrigation have a significantly better visual prognosis. ${ }^{1}$ Immediate treatment of a chemical splash in the eyes should be conducted with the goal of halting continued contact between the ocular surface and the chemical. ${ }^{9}$

Eye wash stations are an important part of keeping employees safe in the workplace. The United States Occupational Safety and Health Administration (OSHA) requires eye wash facilities in workplaces that use corrosive chemicals, research laboratories dealing with human immunodeficiency virus (HIV) and/or hepatitis B (HBV), and any workplace that poses the possibility of an eye splash with $0.1 \%$ or greater formaldehyde. ${ }^{10}$ These stations are also used in other workplaces, including research facilities, laboratories, and other places in which materials could cause injury to the eyes. Eye wash stations are needed in these environments because of the necessity to begin washing an eye within 10-15 seconds after exposure to a hazardous substance to prevent serious, permanent damage to the eyes. ${ }^{11}$

While OSHA regulates what settings require an eye wash station, there are no specifications for installation or operating requirements. The American National Standards Institute (ANSI) created minimum requirements that are followed in order to ensure that eye wash stations are available for proper use when needed. ${ }^{12}$ ANSI standard 358.1-2014 requires that an eye wash station must be located so that an employee can reach it within 10 seconds. It must be positioned 33-45 inches from the floor and be 6 inches from the nearest obstruction. An eye wash must deliver 0.4 gallons per minute for 15 minutes. A combination eye wash/face wash must flush both the eyes and face and has the same requirements for the placement of the station, but must deliver 3 gallons per minute for 15 minutes. Either type of emergency wash station is required to have a valve that activates in 1 second or less and must have a valve that stays open, allowing hands free operation. $^{12}$

In modern eye wash production, water flow occurs from the temporal side of each eye, forming an arc and falling back into the eyewash station basin on the nasal side of each eye. Users put their open eyes into the top of this arc, to keep continuous water exchange without any vertical forcefulness of water flowing directly at the surface of the eye.

Recently, a company has claimed to have created an eye wash station that washes each eye in the opposite direction, with the water flowing from the nasal side of each eye and forming an arc that falls into the basin on the temporal side of each eye. No studies to date have been done to determine if this direction of water flow makes any difference in the effectiveness or comfort of using the eye wash fountain.

The purpose of our study was to determine if the direction of water flow from an eye wash fountain plays a role in the ocular surface reaction to the eye wash procedure and to evaluate subjective responses of comfort when using these differing designs.

\section{Materials and methods}

This study is a prospective open-label study of the effectiveness of two different designs of eye wash fountains by assessing the ocular surface response and surveying subjects following the use of the eye washes. An eye wash station designed with crossed streams of water with each stream originating on the temporal side of the eye (Encon Safety Products, Inc., Houston, TX, USA) was compared to an eyewash station that had water flowing in two diverging paths originating on the nasal side of each eye (Haws, a Traynor Family Enterprise, Sparks, NV, USA). The study was completed under the approval of the Institutional Review Board at The Ohio State University, located in Columbus, Ohio, USA. Subjects provided written informed consent prior to screening.

This study was designed to objectively compare the effectiveness of two different designs of eyewash fountains and subjectively compare the comfort and experience of subjects after using these different designs. Figure 1 describes the study design. Effectiveness of the wash was determined by instilling sodium fluorescein into the tears and assessing its presence or lack of presence after the eyes are washed for two minutes with tepid tap water. A slit lamp examination of the eye was done before and after eye wash exposure. A questionnaire was used, regarding vision and comfort modified from the Ocular Surface Disease Index (OSDI) ${ }^{13}$ with questions added regarding the eyewash itself, including reaction to the direction of the flow of the eye wash and the force of the flow of the eye wash. It should be noted that the diverging stream eye wash station also included a face wash, which we addressed with subjects before surveying them (Figure 1).

\section{Subjects}

To be eligible for the study, subjects were required to be between the ages of 18 and 65 years, were required to have had a comprehensive eye examination in the past 2 years with normal findings on the front surface of the eye, and could not be nursing or pregnant. 


Eye wash visit 1
- Measure BCVA
- Examine ocular surface of eyes
- Use one eye wash for one minute
after instilling NaFI
- Measure NaFI remaining
- Examine ocular surface of eyes
- Measure BCVA
- Complete survey

Inward water flow eye wash

\section{Eye wash visit 2}

- Measure BCVA

- Examine ocular surface of eyes

- Use one eye wash for one minute

after instilling $\mathrm{NaFI}$

- Measure $\mathrm{NaFI}$ remaining

- Examine ocular surface of eyes

- Measure BCVA

- Complete survey
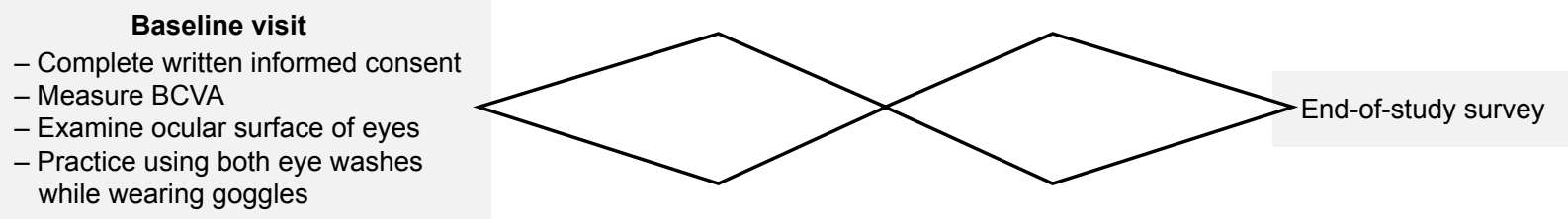

Outward water flow eye wash

Outward water flow eye wash

Figure I Eye wash study design.

Abbreviation: BCVA, best corrected visual acuity.

\section{Study visits}

After consenting to participate in the study, the baseline examination began with a measurement of best correct visual acuity and an examination of the ocular surface of each eye with a biomicroscope. A practice eye wash procedure was done using a pair of goggles designed for teaching people the proper use of an eye wash. Subjects practiced using both of the eye wash stations while wearing the goggles to ensure that they could position themselves correctly and also to make sure they felt they were capable of completing the eye wash study. After determining that subjects were still interested in continuing, subjects were randomized using a randomization table to determine which eye wash station would be used for the first and second study visits. In all cases, the subject wished to begin the first study visit immediately after the baseline visit. At each of the study visits, subjects were given a poncho to wear to protect their clothing from water. The investigator instilled fluorescein using a strip that was wet with eye wash solution and touching it to the lower bulbar conjunctiva with about $2 \mathrm{~mm}$ of touch. Subjects were instructed to hold their own eyelids open with their fingers while their eyes were in the eye wash streams of water. The investigator and coordinator were both present so that one person could measure the time with a stopwatch while both would watch to make sure that the subject was properly using the eye wash and had both eyes in the wash in the best possible alignment. If a subject began to drift away from the eye wash stream, an investigator quickly instructed them to move back into the water stream. After the 1-minute eye wash, the subject was handed a paper towel to dry only their forehead and mouth, but instructed not to touch their eyes. The poncho remained on as we performed a slit lamp examination immediately after using the eye wash. Visual acuity was evaluated again, and a questionnaire was given to the subject. On the second visit, the subject was also given the "End-of-study questionnaire."

\section{Test articles}

The Encon eye wash model number 01040144 was used for water flow originating at the temporal side of each eye. For simplicity, this eye wash is referred to as having "converging flow." This design was compared to the Haws model Axion MSR \#AX13, which has an eye wash flow with streams originating at the nasal portion of each eye, referred to as "diverging flow". The Haws model included a face wash, which necessitated a discussion with each subject to analyze only the eye wash portion of each model.

\section{Statistical analysis}

No previous data, neither experimental nor published, existed upon which to base a sample size calculation. Thus, this attainable sample size was chosen and will be used to project any possible further studies.

For variables that could be treated as continuous, means $\pm \mathrm{SD}$ and paired $t$-tests were used to account for the repeated measurements within subjects. For other variables, 
frequencies and agreement (McNemar's test) were used for comparative purposes.

\section{Results}

This study enrolled subjects aged 20-56 years, with 7 male and 13 female participants.

Twenty subjects were consented and completed the study. All subjects completed their second study visit within 10 days of their first visit.

\section{Safety outcomes}

There were no adverse events in this study.

\section{Visual acuity}

Subjects had normal visual acuity ranging from 20/10 to 20/25-1 when best corrected. There was no significant difference between the change in visual acuity before and after using an eye wash when comparing the converging flow and diverging flow eye washes. Table 1 shows the change in visual acuity when comparing pre- to post-eye wash with each eye wash design. Visual acuity is reported in LogMAR scale with $0.02=1$ letter on the visual acuity chart.

\section{Slit lamp biomicroscope findings}

Subjects had an assessment of the ocular surface before and after using the eye washes. Staining of the corneal surface was recorded at these examinations to determine if the cornea sustained any damage as a result of using the eye washes. There was no real variation in the staining between pre- and post-wash. Table 2 reports the agreement tables for pre- and post-wash by brand and eye.

No clinically significant corneal staining was observed throughout the study.

\section{Efficacy outcomes}

The presence of fluorescein after using each eye wash for 1 minute was considered a primary outcome of effectiveness. Fluorescein was added to the tears immediately before each eye wash. After washing the eyes, the amount of fluorescein remaining in the tear prism at the eyelid margins was graded as none (1), mild (2), moderate (3), or heavy (4). Table 3 shows the comparison of the amount of fluorescein

Table I Change in visual acuity compared as the change from post- to pre-wash with converging flow - the change from postto pre-wash with diverging flow

\begin{tabular}{lll}
\hline Eye & Mean \pm SD & Paired t-test \\
\hline OD & $0.02 \pm 0.08$ & 0.29 \\
OS & $-0.03 \pm 0.10$ & 0.17 \\
\hline
\end{tabular}

Table 2 Corneal staining before and after eye wash use agreement tables

\begin{tabular}{|c|c|c|}
\hline \multicolumn{3}{|c|}{ A Pre- vs post-staining, converging flow OD $(n=20)$} \\
\hline \multirow[t]{2}{*}{ Pre-wash staining } & \multicolumn{2}{|c|}{ Post-wash staining (\%) } \\
\hline & $\mathbf{I}$ & 2 \\
\hline 1 & 95.0 & 5.0 \\
\hline \multicolumn{3}{|c|}{ B Pre- vs post-staining, converging flow OS $(\mathrm{n}=20)$} \\
\hline \multirow[t]{2}{*}{ Pre-wash staining } & \multicolumn{2}{|c|}{ Post-wash staining (\%) } \\
\hline & $\mathbf{I}$ & 2 \\
\hline 1 & 90.0 & 20.0 \\
\hline \multicolumn{3}{|c|}{ C Pre- vs post-staining, diverging flow OD $(n=20)$} \\
\hline \multirow[t]{2}{*}{ Pre-wash staining } & \multicolumn{2}{|c|}{ Post-wash staining (\%) } \\
\hline & $\mathrm{I}$ & 2 \\
\hline I & 100.0 & 0 \\
\hline \multicolumn{3}{|c|}{ D Pre- vs post-staining, diverging flow OS $(n=20)$} \\
\hline \multirow[t]{2}{*}{ Pre-wash staining } & \multicolumn{2}{|c|}{ Post-wash staining (\%) } \\
\hline & $\mathbf{I}$ & 2 \\
\hline 1 & 89.5 & 10.5 \\
\hline 2 & 0 & 5.0 \\
\hline
\end{tabular}

remaining after using the eye wash fountains. There was no significant difference when comparing the average presence of fluorescein after using the Encon and the Haws eye washes. The outward flow eyewash grades were subtracted from the inward flow eyewash scores.

\section{Subjective outcomes}

Subjects were asked to complete a questionnaire (Figure 2) after using each eye wash. Statistical analysis of subjects' responses to these questions found no statistical significance in the differences of the responses between the two eye washes.

The questions in Figure 2 are not responded to in a continuous fashion. In Table 4 are agreement tables to show what changes, if any, occurred. The shaded cells indicate that the responses were the same in both eye wash designs.

\section{End-of-study eyewash questionnaire}

The questionnaire in Figure 3 was given at the conclusion of the study.

Table 3 Presence of fluorescein after eye wash: comparison of converging flow eye wash and diverging flow eye wash (EnconHaws)

\begin{tabular}{lll}
\hline Eye & Mean \pm SD & Paired t-test \\
\hline OD & $-0.42 \pm 0.96$ & 0.07 \\
OS & $-0.16 \pm 0.69$ & 0.33 \\
\hline
\end{tabular}


Do your eyes feel sore?

Not at all (0) Mildly (1) Moderately (2) Severely (3)

Do your eyes feel painful?

Do your eyes feel gritty?

Do your eyes feel light sensitive?

Is your vision blurred?

Comments on any differences felt/experienced between the left and right eyes:

Figure 2 Eye wash study questionnaire.

The results of the questionnaire showed that $70 \%$ of subjects preferred the Encon eye wash for direction of flow. Forty percent of subjects preferred the Encon eye wash for speed of flow while $45 \%$ preferred the Encon eye wash for forcefulness of flow. It should be noted that since these subjects did not actually have anything toxic in their eyes, the questions may have been answered based on the comfort

Table 4 Comparison of survey questions (Encon-Haws)

\begin{tabular}{l}
\hline A Grittiness (n=18) \\
\begin{tabular}{|l|l|l|l|l|}
\hline Encon & & \multicolumn{3}{|l|}{ Haws (\%) } \\
\hline & & I & & $\mathbf{2}$ \\
\hline I & & 83.3 & & 0 \\
\hline 2 & & 5.6 & & 11.1 \\
\hline
\end{tabular}
\end{tabular}

B Light sensitivity $(n=19)$

\begin{tabular}{|l|l|l|l|l|}
\hline \multirow{2}{*}{ Encon } & & \multicolumn{3}{|l|}{ Haws (\%) } \\
\cline { 3 - 5 } & & $\mathbf{I}$ & & $\mathbf{2}$ \\
\hline $\mathrm{I}$ & 78.9 & & 5.3 \\
\hline 2 & & 0 & & 10.5 \\
\hline 3 & & 0 & & 5.3 \\
\hline
\end{tabular}

C Painfulness $(n=18)$

\begin{tabular}{|l|l|l|l|l|}
\hline \multirow{2}{*}{ Encon } & & \multicolumn{3}{|l|}{ Haws (\%) } \\
\cline { 3 - 5 } & & $\mathrm{I}$ & & $\mathbf{2}$ \\
\hline $\mathrm{I}$ & 77.8 & & $\mathrm{II} .1$ \\
\hline 2 & & $\mathrm{II} . \mathrm{I}$ & & 0 \\
\hline
\end{tabular}

D Soreness $(n=19)$

\begin{tabular}{|l|l|l|l|l|}
\hline \multirow{2}{*}{ Encon } & & \multicolumn{3}{|l|}{ Haws (\%) } \\
\cline { 3 - 5 } & & $\mathbf{I}$ & & $\mathbf{2}$ \\
\hline $\mathrm{I}$ & & 47.4 & & 15.8 \\
\hline 2 & 10.5 & & 21.1 \\
\hline 3 & & 0 & & 5.3 \\
\hline
\end{tabular}

E Blurred vision $(n=19)$

\begin{tabular}{|l|l|l|l|l|}
\hline \multirow{2}{*}{ Encon } & & \multicolumn{3}{|l|}{ Haws (\%) } \\
\cline { 3 - 5 } & & $\mathbf{I}$ & & $\mathbf{2}$ \\
\hline $\mathrm{I}$ & 57.9 & & 21.1 \\
\hline 2 & 5.3 & & 5.3 \\
\hline 3 & & 5.3 & & 5.3 \\
\hline
\end{tabular}

Note: The shaded cells indicate that responses were the same between both eye wash designs. of use rather than functionality. Thirteen out of 20 subjects commented that it was more difficult to breathe or to keep eyes in the eye wash while using the Haws face wash, which had diverging water flow.

\section{Discussion}

Little information is published on eye wash safety devices for a comparison of direction of water flow. This study provided some data that show there is not a significant difference in the safety and efficacy of water flow direction when inward and outward flows are compared. This study also provided an opportunity for investigators to observe people using eye wash stations to gather better information on how these devices work and how people interact with the eye wash fountain during its use.

In our study, subjects were instructed to hold their eyes open with their fingers, as is suggested with eye wash station usage. This aspect of using the device was not difficult, despite the fact that the literature says that another person may be needed to assist with this in a true chemical accident. ${ }^{6}$ One aspect of using the eyewash station that proved difficult for subjects was breathing while using the eyewash, whether real or perceived by the subject. The breathing difficulty was reported more frequently when using the eye wash station that also included a face wash. Subjects who were of smaller statute had difficulty keeping their eyes in the eye wash while the water of the face wash was going into their nose. Taller subjects did not have as much difficulty. Several subjects said that they tried to hold their breath during the eye wash, with both water flow directions, and surmised that

\section{Do you prefer the first or second eye wash for:

\begin{tabular}{lll} 
Direction of flow & Eye wash 1 & Eye wash 2 \\
Speed of flow & Eye wash 1 & Eye wash 2 \\
Forcefulness of flow & Eye wash 1 & Eye wash 2 \\
Comments as to why: & & \\
\hline
\end{tabular}

Figure 3 End-of-study eye wash questionnaire. 
it was possibly an instinct due to the perception of "being under water." With 13 out of 20 subjects commenting on difficulty breathing with the face wash component, it raises the question of whether or not the face wash component would be helpful if keeping the eyes in the eyewash stream for a much longer period than 1 minute. Certainly washing the face may be needed, but if those streams of water prevent a person from keeping their eyes in the water stream, a stand-alone eye wash may be more likely to wash an eye thoroughly on its own.

During the study, investigators observed that subjects who were shorter or those who were overweight had difficulty approaching the eye wash from above and complained more of getting the water of the face wash in their nose and mouth. They were also more likely to try to wash their eyes with the streams of the face wash, rather than the eye wash. Our subjects were all quickly corrected if they drifted into the face wash water streams instead of the eye wash streams, but it may be difficult for them to reach or know if they are in the proper stream of water in an emergency situation. While tall people may eventually get tired from leaning over for long periods of time, they are able to align themselves from above regardless of the height of the eye wash.

The other observed difficulty with using the eye wash stations centered on keeping the eyes in the actual streams of water. During our study we were constantly watching and coaching subjects to stay in the water so that they would not drift away from the streams of water. In the case of a chemical burn, hopefully another person could assist with directing the eyewash user to stay in the water. Unfortunately, one feature of the eye wash fountain with diverging water flow was that only one exact position would keep both eyes in their respective stream of water at one time. Because the streams of water on the eye wash with converging flow crossed each other, there was a larger area over which both the eyes could be washed simultaneously.

\section{Influence of inter-pupillary distance on eye wash use}

Since investigators observed some subjects having difficulty keeping their eyes in the flow of water of the eye washes, further observations of the devices were made. An eye wash station consists of two streams of water that arc and then plateau at each peak, creating a stream of water that does not exert vertical force upon the eye as clean water is constantly exchanged. While it is initially assumed that the flow of the eye wash may influence the direction of tear and toxin flow, the eyes are ideally placed in the position at the top or peak of water flow from the spigot so that eyes are rinsed without force being applied to the eye. This study did not evaluate the actual direction of tear flow to determine if tear flow continues in the normal flow pattern through the puncta. The converging flow eye wash has two crossed streams of water which creates one peak or horizontal plateau of water in which to place both eyes. The plateau created by the converging streams is approximately $120 \mathrm{~mm}$ wide, allowing both eyes to be washed simultaneously for users with an inter-pupillary distance of $120 \mathrm{~mm}$ or less. The diverging eyewash has two streams creating two separate horizontal plateaus of water, one for each eye. The plateaus or areas of the streams which are at the peaks, are considerably farther apart when compared to the distance between eyes in most people. From the center of one peak to the other, the distance is $110 \mathrm{~mm}$. The smallest distance between these peaks, when the water stream is just about to peak, is about $75 \mathrm{~mm}$. The average inter-pupillary distance (IPD) is $64.0 \mathrm{~mm}$ for males or 61.7 $\mathrm{mm}$ for females. ${ }^{14}$ Because the average IPD is less than the distance between the two plateaus created by the diverging flow eye wash, it may be difficult to wash both eyes equally at the same time. Figures 4 and 5 are photographs of the water flow of each device.

The manufacturer of the diverging flow eyewash uses the fact that water flowing toward the outer part of the eye is "the standard healthcare protocol," preventing an eye wash from sweeping contaminants toward the inner corners of the eyes. ${ }^{15}$ An extensive literature shows that emergency room protocol for washing an eye is done by washing or dripping water or a solution from the inner part of the eye while the patient is positioned so that the solution drips toward the outer part of the eye. This is likely done so that the eye wash solution used does not run into the opposite eye, and no research found in our literature search discusses any specific reason for this protocol.

\section{Conclusion}

In this study there was no significant difference in the efficacy of an eye wash fountain when comparing converging and diverging streams of water. Observations of the measurements of the water flow positions and their relationship to the distance between most eyes make an eye wash with converging streams of water appear more likely to wash both eyes simultaneously in a greater population of people. 


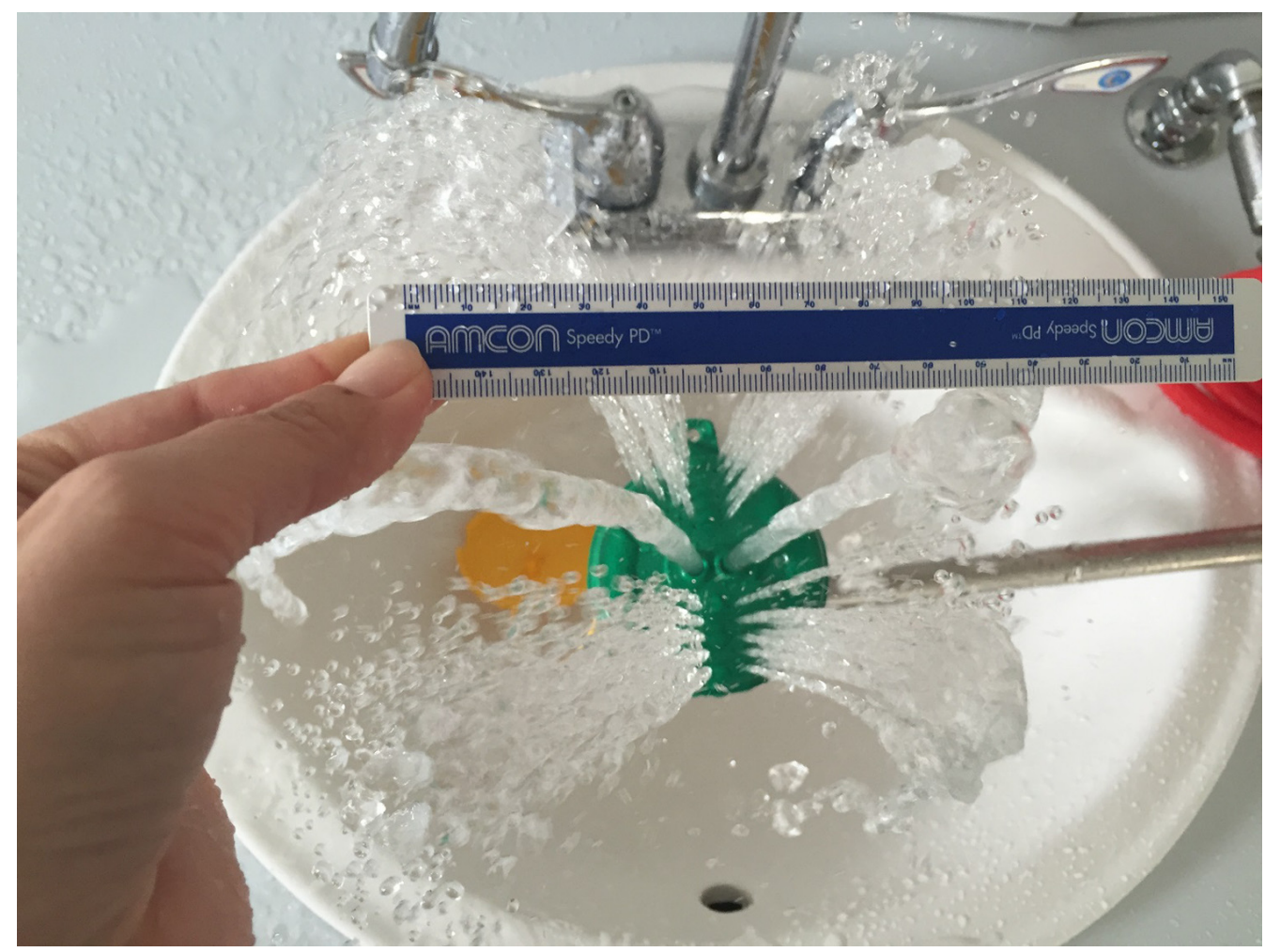

Figure 4 The water flow of an eye wash station with diverging water flow. This model includes a face wash flow of water above and below the eyes. The distance between the peaks of water flow are approximately $110 \mathrm{~mm}$ apart.

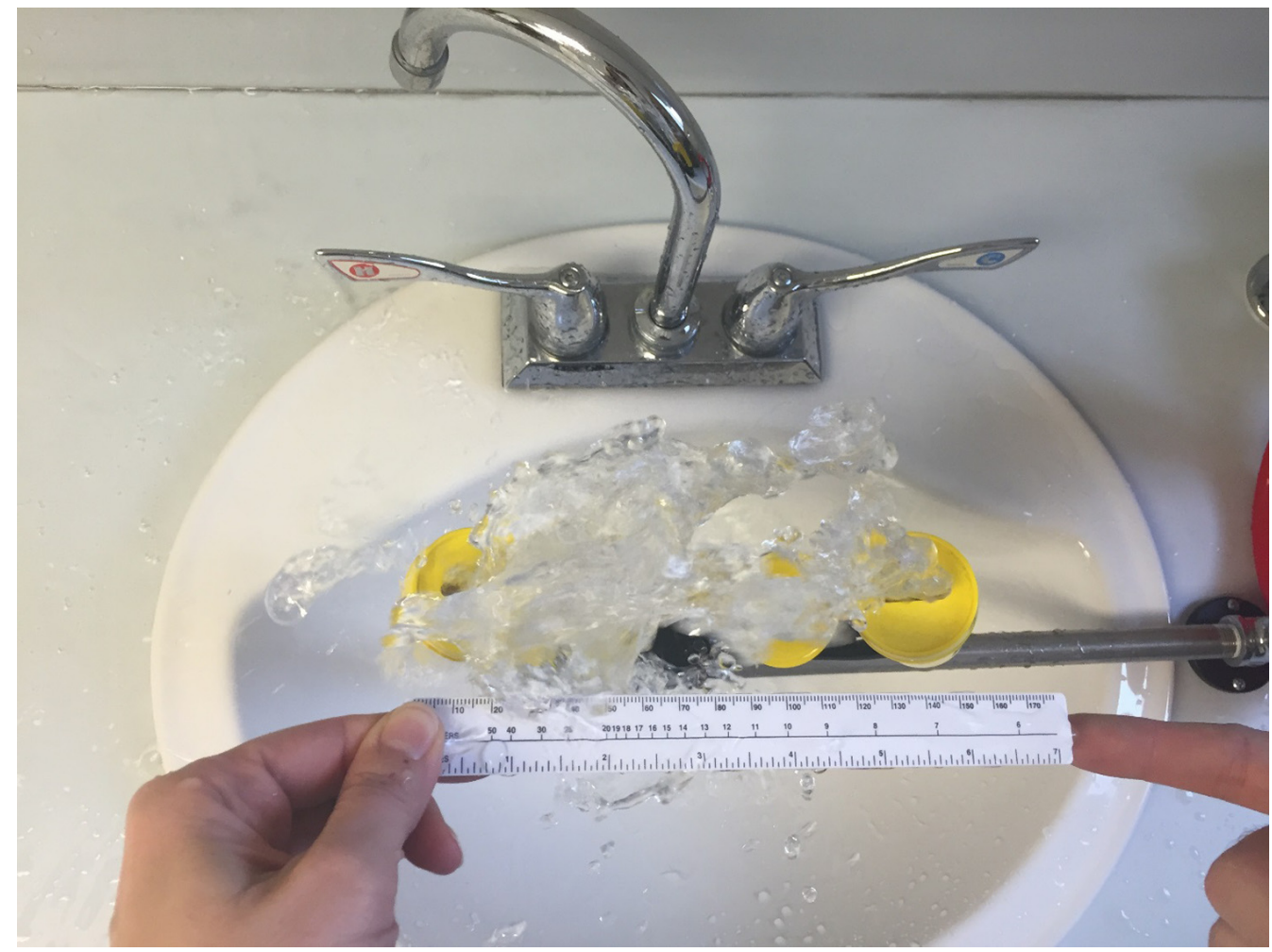

Figure 5 The water flow of an eye wash station with converging water flow. The two streams of water form one large plateau of water that will accommodate people with an inter-pupillary distance of $120 \mathrm{~mm}$ or less. 


\section{Acknowledgments}

This study was completed with funding from Encon Safety Products, Inc. We would like to acknowledge Matthew Kowalski for his assistance in compiling data.

\section{Disclosure}

The authors report no conflicts of interest in this work.

\section{References}

1. Kuckelkorn R, Schrage N, Keller G, Redbrake C. Emergency treatment of chemical and thermal eye burns. Acta Ophthalmol Scand. 2002; 80(1):4-10

2. Singh P, Tyagi M, Kumar Y, Gupta KK, Sharma PD. Ocular chemical injuries and their management. Oman J Ophthalmol. 2013;6(2):83-86.

3. Haring RS, Sheffield ID, Channa R, Canner JK, Schneider EB. Epidemiologic trends of chemical ocular burns in the United States. JAMA Ophthalmol. 2016;134(10):1119-1124.

4. Hoffman RSNL, Howland MA. Goldfrank's Manual of Toxicologic Emergencies. Blacklick, OH, USA: McGraw-Hill Professional Publishing; 2007.

5. Palao R, Monge I, Ruiz M, Barret JP. Chemical burns: pathophysiology and treatment. Burns. 2010;36(3):295-304.
6. Kuckelkorn R, Kottek A, Schrage N, Reim M. Poor prognosis of severe chemical and thermal eye burns: the need for adequate emergency care and primary prevention. Int Arch Occup Environ Health. 1995;67(4): 281-284.

7. Schrage NF, Langefeld S, Zschocke J, Kuckelkorn R, Redbrake C, Reim M. Eye burns: an emergency and continuing problem. Burns. 2000; 26(8):689-699.

8. Lewis CJ, Al-Mousawi A, Jha A, Allison KP. Is it time for a change in the approach to chemical burns? The role of Diphoterine ${ }^{\circledR}$ in the management of cutaneous and ocular chemical injuries. $J$ Plast Reconstr Aesthet Surg. 2017;70(5):563-567.

9. Wagoner MD. Chemical injuries of the eye: current concepts in pathophysiology and therapy. Surv Ophthalmol. 1997;41(4):275-313.

10. United States Occupational Safety and Health Administration information Sheet 3818. 2015.

11. Canadian Centre for Occupational Health and Safety Fact Sheet: Emergency Showers and Eyewash Stations. 2015.

12. ANSI/ISEA. American National Standard for Emergency Eyewash and Shower Equipment. Z358. 2014.

13. Schiffman RM, Christianson MD, Jacobsen G, Hirsch JD, Reis BL. Reliability and validity of the Ocular Surface Disease Index. Arch Ophthalmol. 2000;118(5):615-621.

14. Gordon CC, Blackwell CL, Bradtmiller B, et al. 2012 Anthropometric Survey of U.S. Army Personnel: Methods and Summary Statistics. Yellow Springs, OH 45387. 2014.

15. Haws Corporation. Axion MSR ${ }^{\circledR}$ Brochure. 2014.
Clinical Ophthalmology

\section{Publish your work in this journal}

Clinical Ophthalmology is an international, peer-reviewed journal covering all subspecialties within ophthalmology. Key topics include: Optometry; Visual science; Pharmacology and drug therapy in eye diseases; Basic Sciences; Primary and Secondary eye care; Patient Safety and Quality of Care Improvements. This journal is indexed on

Submit your manuscript here: http://www.dovepress.com/clinical-ophthalmology-journal

\section{Dovepress}

PubMed Central and CAS, and is the official journal of The Society of Clinical Ophthalmology (SCO). The manuscript management system is completely online and includes a very quick and fair peer-review system, which is all easy to use. Visit http://www.dovepress.com/ testimonials.php to read real quotes from published authors. 